\title{
Flexible Software Architecture for User- Interface and Machine Control in Laboratory Automation
}

BioTechniques 25:698-705 (October 1998)

\author{
Ethan B. Arutunian, Deirdre \\ R. Meldrum, Neal A. Fried- \\ man and Stephen E. Moody ${ }^{1}$ \\ University of Washington, \\ Seattle and ${ }^{1}$ Orca Photonic \\ Systems, Redmond, WA, USA
}

\section{INTRODUCTION}

Biologists and laboratory technicians often struggle with inadequate user-interface designs for complicated instrumentation. Common approaches to user-interface design include small liquid crystal displays (LCDs) with keypad or keyboard inputs and dedicated computers with command-line user interfaces $(2,4,5)$. In both cases, usability is consistently sacrificed by requiring user knowledge of cryptic commands or lengthy written scripts.

For more sophisticated systems featuring graphical user interfaces (GUIs), a specific computer platform must be chosen before user-interface development. Although GUIs are generally easy to use, this solution is inflexible for both users and developers. First, manufacturers may be forced to ship the chosen platform with the system. Second, future development must continue to support the platform. Finally, the laboratory receiving the system must be familiar with the platform, and the system must integrate well in the laboratory.

Thus, existing user-interface architectures could not meet the functional needs of the laboratory instruments under development by our group at the University of Washington (Seattle, WA, USA). The existing approaches did not offer the right combination of capability, ease-of-use, flexibility and rapid development that our projects demand. To overcome these limitations, we have developed a user-interface architecture that exploits the open, standards-based culture of the Internet, while retaining capability to develop a fully interactive GUI. This design allows for use of existing source code and protocols, mini- mizes development time, maintains platform independence and extends flexibility. It is generally applicable to all automated laboratory instrumentation. Here, we demonstrate it on the Acapella, an automated fluid-sample-handling system that is currently under development at the University of Washington.

\section{SYSTEM REQUIREMENTS}

The motivation for a new user-interface system architecture stems from the immediate application: Acapella, an automated fluid-sample-handling system (6). This system is being developed by the Genomation Laboratory at the University of Washington, Seattle for use in genomic applications, including: sequencing reactions, restriction enzyme digests, polymerase chain reactions (PCRs) and screening reactions. Individual samples are aspirated from a standard 96-well or 384-well microplate into glass capillaries; reagents are then added with piezo-ceramic dispensers. The resulting solution is mixed and output into trays that are compatible with thermal cyclers and gel loaders. A specific goal of the project is to achieve very high sample throughput: 1000 samples/8 h in the first prototype; 5000 samples/8 $\mathrm{h}$ in a second-generation machine currently under development.

The nature of both the machine and its development places a number of direct requirements on the user-interface architecture. First, the machine is still evolving; therefore, any user-interface must be flexible and allow for rapid adjustment as the instrument itself develops. Second, the user interface must be extremely user friendly to offer users the 
capability to request very complex processing protocols. Principal user tasks include designing complicated experiments, monitoring the progress of processing once initiated and, once processing is complete, logging and analyzing detailed "history" data in a secure but accessible fashion. The interface should allow users to accomplish these tasks quickly, flexibly, with minimum error and without extensive training.

Furthermore, the goals of the project intrinsically require an interface that gracefully serves multiple users, since Acapella is intended to become a laboratory-level facility that can service the needs of a widely distributed network of users. A remotely accessible user interface allows users to interact with the machine without requiring physical proximity. Similarly, multiple users must be protected from interference with each other as they define and run their experiments, since samples belonging to different users can be processed by the machine within a short period of time.

Lastly, the "user-facility" nature of the instrument requires segregation of user and machine management functions (although some or even many users will also be system managers). Users should have an interface that flexibly allows them to define what they want done, while machine managers should be able to determine how the machine can best be configured to meet those needs.

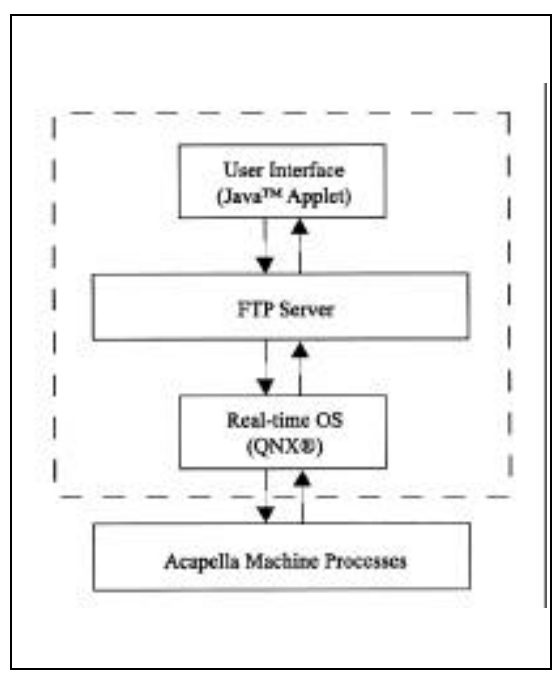

Figure 1. Hardware architecture.

\section{METHODOLOGY AND ARCHITECTURE}

Careful design of overall system architecture was essential to meet the defined project goals. For Acapella, the adopted architecture consisted of three distinct layers, as shown in Figure 1. The user/manager workstation, machine-control processor and individual hardware input/output (I/O) layers each have well-defined functions. As a result, the hardware and software for each layer can be selected to optimally perform just one element of the total task. The remainder of this paper focuses on our implementation of this architecture and the mechanisms for communication between the user-interface layer and the dedicated hardware in the lower layers.

The software architecture (Figure 2) can be described most easily by starting from the center (machine control) layer. The machine controller is responsible for the overall real-time machine control and operation. This controller be- gins with a description of sample-processing requirements as defined by users and is responsible for all detailed machine actions needed to complete the user prescriptions until finished samples are delivered to the machineoutput stream. The machine controller is a server to the user-interface layer and a client to the local-processor layer.

The user-interface layer consists of graphical client software running on any number of remote computers. Each client communicates downward to the machine controller using standard protocols described below. This layer constitutes the interface that allows users to define sample-processing prescriptions and managers to define machine configuration. This information is then downloaded to the machine controller, where it is stored for later use. Views of the current machine and sample status, as well as sample history, can be uploaded to the interface layer for viewing.

The lowest level of the architecture consists of a series of very small

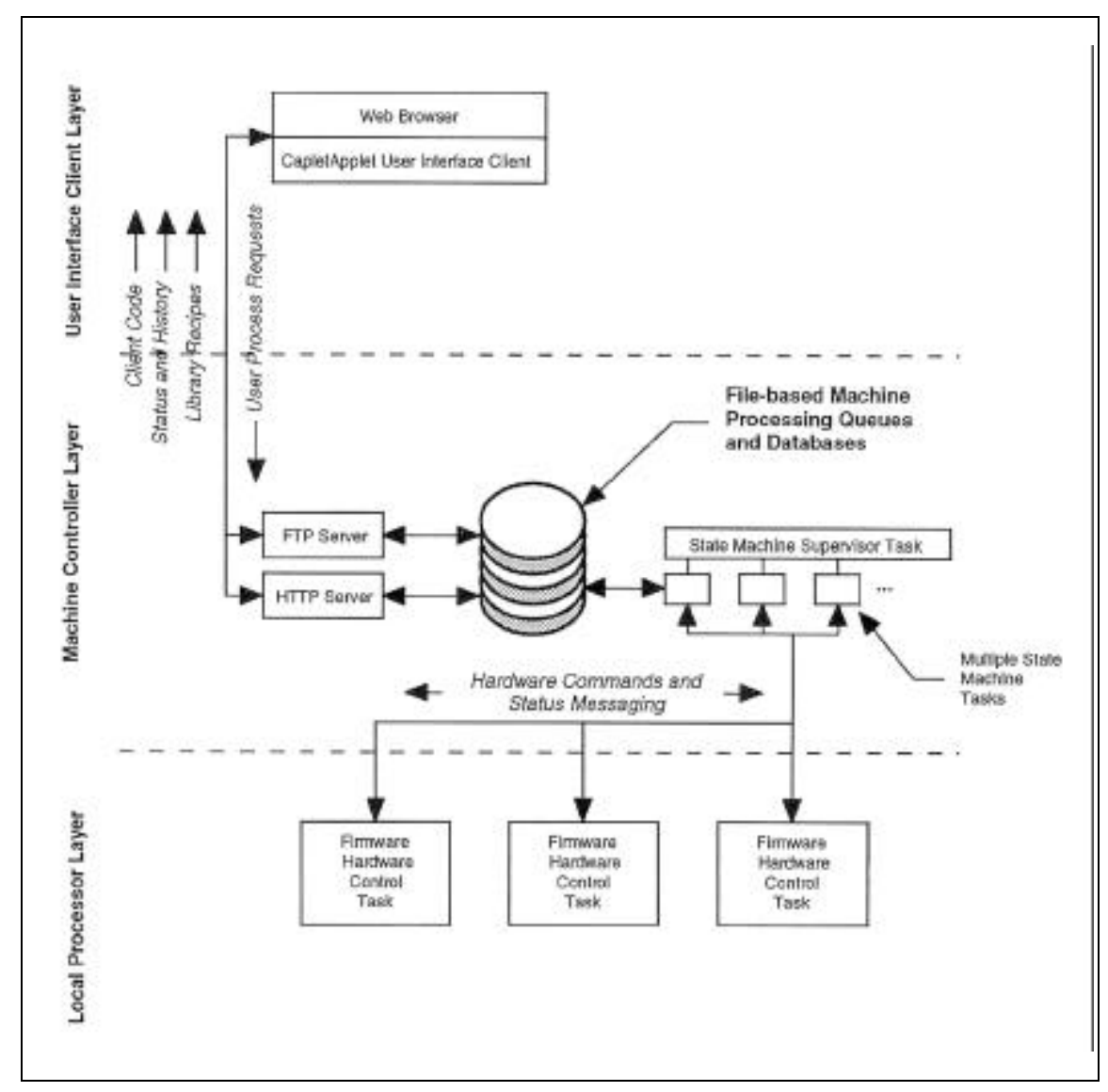

Figure 2. Software architecture and information flow. 
processors (three single-board computers) that interface directly to machine hardware. Each processor is responsible for controlling one or more of the hardware subsystems. Because of the low cost of these processors, it was not a design goal to minimize their number. The processors communicate with the machine controller by passing relatively high-level messages over an RS232 communication line. In this design, the local processors act as servers to the machine controller, providing specific hardware functions in response to request messages from the machinecontroller client. Use of these "satellite processors" provides the ability to control individual subsystems during development and negates the need to develop device drivers for all of the specialized hardware required for our control problem.

This architecture has been implemented on Acapella, and it has worked well as a prototype model for this project. Some of the benefits and costs that we have observed follow. The most significant cost is the "hardware-intensive" character of the resulting control system design. It is undeniable that Acapella could be implemented with considerably less computing hardware using a different architecture. Given the low cost of this hardware and the benefits gained from reducing development labor, we view this issue as more aesthetic than real. It is certainly possible that "hardware optimization" could become an issue in a commercialized implementation of the system in the future.

The benefits of the design are many; most can be attributed to the modular architecture. (i) The separation of machine control from user interface has allowed development of these two processes to proceed independently. (ii) Designers have been free to adopt optimum hardware and software operating environments for each layer. For example, the user-interface layer can run on familiar platforms like Windows ${ }^{\circledR}$, while the machine controller runs under a high-capability, real-time operating system. (iii) The low-level, multiple-processor architecture has several advantages: it has allowed for parallel processing of hardware subsystem jobs and has made possible the simultaneous development of different hardware subsystems.

\section{IMPLEMENTATION ISSUES}

Key design decisions of the software implementation include the following. (i) The notion of an exchange of small files through a remote file system has been used as the architecture for communication between user clients and the machine-control server. (ii) Standard Internet protocols and methodologies have been used to the greatest extent possible. This has resulted in ready inter-operability between different platforms, access to public-domain code for many of the communication components and use of the full Internet/IP physical infrastructure for communication between users and the machine. It has also enabled software development to avoid proprietary protocols. (iii) A decision was made early in development to adopt a fully capable real-time operating system (RTOS) at the machine-control layer. Although the total processing throughput required by Acapella is not extreme, the use of a true RTOS has permitted integration of a multitude of functions at the machine-control layer (by segmentation into independent, concurrent processes) without compromising the responsetime needs of the machine hardware.

\section{Remote File System}

Users design sample processing jobs off-line using the user-interface client. These jobs are then downloaded to the machine-control layer as small files, which are entered into a queue of "pending" user requests. The queue simply consists of a designated subdirectory within the machine controller's file system. Job files are deposited in the queue directory by the client using the remote file system. On the machine-controller side, the only software necessary to implement this function is a standard file-server process.

At a system operator's request, the master machine-controller process scans the queue directory and chooses a job file for processing. The token job file is then moved into an "active" directory and finally to a "finished" directory once the requested processing is complete. The client user interface is able to monitor and display the status of this progression simply by requesting directory lists of the designated subdirectories using the remote file system facilities.

This file-oriented architecture delivers a number of important functions with no additional coding. For example, job files in the various subdirectories are properly tagged with the individual user's identity (the owner). Each job file is protected with permissions associated with each owner. Similarly, the user-interface client can put a "pending" file on hold by simply changing specific attributes of the file's permissions. As a job is processed by the system, information pertaining to each individual sample is appended to the job file and moved through the directory structure seamlessly. This allows users to monitor each sample as it passes through the system or collectively after the job has finished. This method also facilitates the integration of a database system with sample results further downstream.

The remote file system capability has been developed using the standard Internet File Transfer Protocol (FTP). File transfers between client and server (machine controller) are accomplished by integrating an FTP client into the userinterface and running a system-wide FTP server on the machine controller.

\section{Machine Controller}

The machine controller manages the hardware-specific control code and operates under a deterministic real-time operating system. In addition, the machine-control layer also acts as a server for the HyperText Transfer Protocol (HTTP) and for our remote file system FTP protocols. These standard, open protocols provide the key transfer mechanisms to effectively link the userinterface and machine-control layers. HTTP is used to serve the user client with the user-interface application, in the form of World Wide Web (WWW) pages and embedded Java ${ }^{\mathrm{TM}}$ applets, as described below. A modified FTP server process is used to transfer sampleprocessing instructions and status reports between the user-interface and machine-controller layers.

We have selected the QNXTM operating system (QNX Software Systems, Kanata, ONT, Canada) for the machine-control processor. QNX is a small, fast and reliable deterministic 
real-time operating system. QNX includes a standard Unix ${ }^{\circledR}$ file system, which is important to our FTP/remote file system approach. For Acapella, QNX is installed on "industrial PC" hardware, running comfortably on a 66 $\mathrm{MHz} 80486$ with 16 Mbytes of random-access memory (RAM).

QNX also implements standard Unix programming interfaces and thus provides all of the usual Unix utilities and network facilities. More importantly, an enormous store of publicly distributed Unix software can be readily (and inexpensively) ported to this platform. In our case, we run the freely distributed Apache server to provide HTTP services and a modified version of the publicly distributed Washington University FTP server (WU-FTP) to provide the remote file system interface needed to service the user-interface client (1).

The machine-control software is implemented as a series of cooperating tasks that operate specific subsets of the machine hardware. These multiple machine-control tasks are implemented in a finite-state-machine (FSM) paradigm. The state machines are represented within a simple state-machine language and run on top of a general FSM support framework that was written for this project. The multiple-state machines are synchronized and coordinated by an "intermachine" communication protocol that is written into the FSM infrastructure using QNX interprocess communication mechanisms. More discussion of the FSM system will be available in a forthcoming publication (Osiris Business Systems, Ann Arbor, MI, USA).

The interface to hardware is provided by a serial of small, inexpensive single-board computers (Z-world Engineering, Davis, CA USA). Code for these processors is developed on a PC in $\mathrm{C}$ programming language and downloaded to the boards, where it is retained in battery-backed static randomaccess memory (SRAM).

\section{User-Interface Implementation}

The original goals of the user interface included the desire to achieve platform independence for the user-client software. Ideally, users should be able to work effectively from their existing laboratory and desktop machines, with no special software installation or platform restrictions. The common laboratory environment contains a diverse mixture of Windows, Macintosh ${ }^{\circledR}$ and Unix machines, and TCP/IP networking is nearly universal.

This requirement led naturally to a decision to implement the user client in Java (Sun Microsystems, Palo Alto, CA, USA) (3). Java is a C++-style programming language that is inherently cross-platform, since it executes on a "virtual machine" that has been implemented on a full range of parent platforms. Originally conceived as an embedded systems language, Java is now widely used as a means for extending the static HyperText Markup Language (HTML) capabilities of the WWW. Java provides a mechanism for downloading "applets" embedded within HTTP WWW pages, so that moderately large and fully interactive applications can be delivered to the user with no explicit installation on the user's machine. In this case, a user need only enter the appropriate uniform resource locator (URL) into any standard Internet browser to download the user-interface applet (embedded within a container HTML page) from the Web server operating on the machine-control processor.

We chose this approach in preference to a more straightforward implementation using HTML, forms and Common Gateway Interface (CGI) programs to allow for the implementation of a fully featured, responsive and interactive graphical interface. Java provides a fairly sophisticated graphics toolkit for applets, allowing designers to customize the look and feel. The user interface designed for this project is oriented around the image of a 96-well microplate. Users are able to select wells in the plate and apply sample-processing instructions using familiar "pointand-click" operations. Figure 3 shows a screen capture of the user interface, as acquired from an actual operating example of the user-interface client. A demonstration version of this applet can be found on our Web page (7).

Java also contains an array of classes to implement network-oriented client/server operations, including an FTP client. Unfortunately, the specifics of Java applet security forced us to move outside the standard FTP protocol to meet our functional requirements. There are two major networking restrictions placed on Java applets for security reasons. (i) An applet can only open a client connection to the same node from which the applet was served. (ii) A Java applet may not open a server, or listener, socket on the client machine. Both restrictions are intended to prevent rogue applets from making connections (in either direction) with "third-party" machines and possibly exchanging privileged or destructive data without user knowledge or consent.

The restriction in applet-listener function proved to be a significant barrier to our "pure standards" implementation. The standard FTP implementation actually opens two connections between client and server, one in each direction. Unfortunately, Java applet security forbids such a transaction. To overcome this limitation, we modified both the Java FTP client and the WUFTP server so that connections are only established in the allowed direction. This modified protocol is viable within the existing Java applet security paradigm. We emphasize, however, that the need to modify the FTP protocol is imposed by our decision to implement the client in Java and not by our choice of system architecture. Both the Java client and modified FTP server, along with a description of the problem, are available on our Web site (7).

\section{User-Interface Description}

The following discussion provides the reader with a clear understanding of the actual use of the Acapella user interface as implemented. After entering the appropriate URL into a Java-capable browser, users are presented with a "Log On" prompt. Logging on requires the user to enter a name and password for an account on the Acapella system. The applet then verifies this information by attempting to establish a modified FTP connection to the machine's FTP server. If successful, the user is presented with a list of previously designed trays and the option to create, edit or view one or more of them. At this point, users can also monitor the queue of pending trays already downloaded to the machine.

When a user chooses to create or 
edit a tray, a dialog window appears (similar to the one shown in Figure 3). A schematic representation of a 96well microplate, capillary output cassette and list of existing recipes are visible. Users select wells in the microplate in the same manner as one selects cells in a spreadsheet. Reactions are specified by selecting a recipe from the list and "applying" it to the selected wells with a single action. The capillary output cassette is automatically updated to illustrate the actual sequence of reactions that will be executed when the machine is run.

Users can create or edit recipes easily. Requested aspiration volume, reagent volumes for each dispenser on the machine and mixing profiles together comprise individual recipes. Future recipes will include thermal cycling protocols as well. Recipes can be combined into "books" to generate multiple individual reactions from a single microplate well (e.g., dye-primer sequencing reactions).

As users create and modify trays and recipes, the new protocols are downloaded to the machine server for storage. These files are protected with the user permissions established at log on. Users can also submit these files to the job queue for processing. Once submitted, job files appear on an LCD panel on the machine. Technicians use this facility to select and execute appropriate jobs based on priority and current machine configuration. Finished jobs are removed from the job queue automatically and stored on the machine server.

A demonstration version of the userinterface applet can be found at our Web site (7).

\section{DISCUSSION AND SUMMARY}

A rich and flexible control system and user-interface architecture was implemented for Acapella, an automated fluid-sample-handling system. In our ongoing development of the Acapella system, we have demonstrated that this new system architecture provides an effective environment for controlling an evolving high-level instrument. In particular, the concept of modular, layered control and interface functions, combined with a remote file system mechanism to couple the two, has allowed us to implement a complex client/server application with very little coding.

Actual control of the Acapella instrument is implemented on QNX, a realtime operating system with Unix application programming interfaces. Even when running on very modest hardware, this operating system has given us both excellent real-time performance and access to free HTTP and FTP servers.

Our first user-interface client implementation is as a Java applet. This approach provides a high-level graphical user interface and, assuming a standard Java-compatible Web browser is available, allows users to work from any connected workstation, independent of platform. Unfortunately, the Java client has been less successful than we had hoped because of factors that cannot be avoided within the current state of Java implementation. First, Java's applet security implementation required a modification to the FTP server that provides the link between the applet and the QNX file system. This forced us to compromise our "pure Internet standards" design goal. Second, the "cross-platform" claims made for Java have proven, in our opinion, to be somewhat exaggerated. We have discovered numerous inconsistencies and incompatibilities between Java implementations on different client platforms, and even on different browsers, as we have attempted to deploy the applet client across a variety of user desktop computers.

We emphasize that Java client implementation is not a core part of our architecture. It is entirely possible to implement a client to our existing server implementation in any language, on any platform. It is even feasible to support multiple client implementations concurrently for different functionality or different user communities. The only requirement is that the client platform support the underlying Internet protocols. This ability to separate (both in time and in process) user-client development issues from machine-control development is probably the greatest

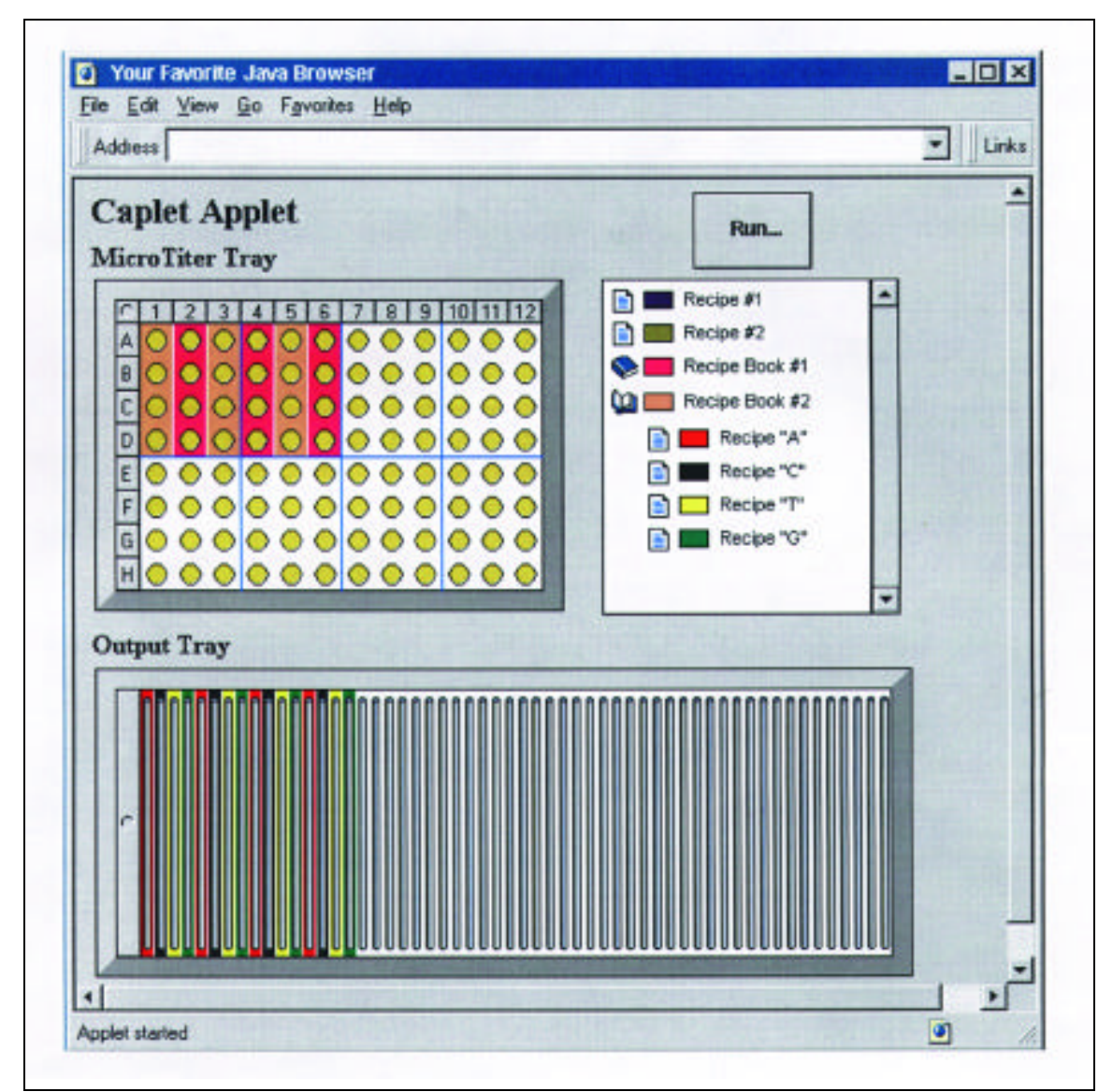

Figure 3. The graphical Java applet user interface. 
single strength of the client/server approach that we have adopted.

User interfaces are an important but often neglected aspect of laboratory automation. We have successfully designed an interface for an automated fluid-sample-handling system that meets the ambitious goals established during the initial machine design. A careful design of overlying system architecture, with a strong division between different kinds of functions, has been the key to a successful result. This segmentation provides easier (and faster) system development, greater flexibility and faster execution. Once the architecture was defined, the necessary components were derived from readily available software resources: $(i)$ QNX, a fast and compact real-time operating system, (ii) Java, which provides tools to easily develop a platform-independent user-interface, open Internet standards and tools, such as the Apache Web Server, and (iii) a modified FTP server based on public-domain code.

\section{ACKNOWLEDGMENTS}

The authors thank the National Institutes of Health (NIH) National Human Genome Research Institute for the support of this research on Grant No. 1 R01 HG01497-01. We also thank Drs. Jean Peccoud and Harold Evensen for their suggestions for improving this manuscript.

\section{REFERENCES}

1.Apache Web Server Project. Code and documentation [Internet]:http://www.apache.org

2.Boyd, J.C., R.A. Felder and J. Savory. 1996.

Robotics and the changing face of the clinical laboratory. Clin. Chem. 42:1899-1900.

3.Carrier, M. 1997. Java with an edge. Object Magazine 7:50-53.

4.Chou, D. 1996. Integrating instruments and the laboratory information system. Am. J. Clin. Pathol. 105:S60-64.

5.Markin, R.S. 1992. Laboratory automation systems: an introduction to concepts and terminology. Am. J. Clin. Pathol. 98:S3-10.

6.Meldrum, D.R. 1997. A biomechatronic fluid-sample-handling system for DNA processing. IEEE/ASME Transactions on Mechatronics 2:99-109.

7.The Acapella Home Page. [Internet]:http:// gnl.ee.washington.edu/gnl/acapella
Received 9 October 1997; accepted 24 June 1998.

Address correspondence to:

Dr. Deirdre Meldrum

Department of Electrical Engineering

University of Washington

Box 352500

Seattle, WA 98195-2500, USA

Internet:deedee@ee.washington.edu 